\title{
Theoretical Analysis of Unsteady Characteristics of Marine Propeller in Ship's Wake
}

\author{
by Bakri Mohammed Idris*, Member Hajime Maruo**, Member \\ Mitsuhisa Ikehata**, Member
}

\begin{abstract}
Summary
The highly loaded marine propeller is affected seriously by non-linear effects of propeller race and hydrodynamic lift. The vortex lattice lifting surface model proposed by Kerwin seems to be the most suitable to deal with such non-linear effects. This Kerwin's model has been applied to develop the theoretical method for calculating the unsteady characteristics of a propeller in the harmonic wake in the previous paper by a part of the authors and another researcher. In the present paper the same methodology is employed and extended to a more general form for theoretical analysis of unsteady propeller characteristics in ship's wake, and some numerical results by this method are compared with those of other linearized theory and with experiments.
\end{abstract}

\section{Introduction}

The unsteady characteristics of marine propellers can be predicted using a computer program based on the lifting surface theory in today's design practice. Such predictions are available with satisfactory accuracy in case of the moderately loaded condition, but they become less accurate in case of highly loaded condition, because most employed lifting surface theories are in the category of the linearized theory. On the highly loaded propeller there exist serious nonlinear effects such as deformations including rollup of trailing vortex sheets, the contraction of the slip stream and the second order term of hydrodynamic lift. The vortex lattice lifting surface model proposed by Kerwin seems to be the most suitable to deal with such non-linear effects ${ }^{1)-4)}$. A part of the authors and another researcher have developed a method to calculate the unsteady characteristics of marine propellers in a non-uniform flow of the simple harmonic wake employing Kerwin's deformed vortex model ${ }^{55,6)}$. The ship's wake can be expanded in Fourier series on a circle in the propeller disk by means of the harmonic analysis. Since each component of such Fourier series is a harmonic wake, such theoretical method previously developed for the

* Graduate School of The University of Tokyo (He was a student of master cource of graduate school of Yokohama National University during this research work.)

** Yokohama National University simple harmonic wake can be applied to each harmonic component of ship's wake. To sum up all solutions of circulation of vortices to harmonic components of ship's wake will give us the resultant circulation of vortices. Then, one can calculate unsteady hydrodynamic forces of marine propellers from Kutta-Joukowski's and other theorems. Thus, a method for theoretical analysis of unsteady characteristics of marine propeller in ship's wake is developed in this paper, and the numerical results calculated by this method are compared with those calculated by Koyama's method ${ }^{7), 8)}$ which is based on the linearized theory of Hanaoka9) or with experimental results.

\section{Theory and Calculation}

\subsection{Fundamentals of Method}

Under fundamental assumptions of sufficiently thin blades, rotating with a constant angular velocity about a common axis in an unbounded fluid and for the purpose of digital computation, the propeller blades can be represented by the distribution of source and vortices lying on the mean camber surface of each blade, together with a distribution of vortices shed into the wake. Therefore, the vortex distribution on the blade is composed of "spanwise" and "chordwise" components, while the corresponding components in the wake are termed "shed" vorticity and "trailing" vorticity.

The application of Kelvin's theorem for the conservation of circulation makes it possible to express all components of the vortex system in terms of the time history of the distribution of 
spanwise vorticity. This leaves the spanwise vorticity as the unknown function of time and space to be determined from the boundary condition of the flow.

The propeller is considered to be operating in a known inflow field representing the wake field of the ship. The components of the inflow wake field are customarily specified only in the plane of the propeller, and it is assumed that their variation over the axial extent of the propeller can be ignored.

\subsection{Mathematical Modeling of Propeller System}

Kerwin's deformed wake model is employed because the non-linear effects of contraction and roll-up of trailing vortex sheets can be taken into account.

Fig. 1 illustrates the deformed wake model with
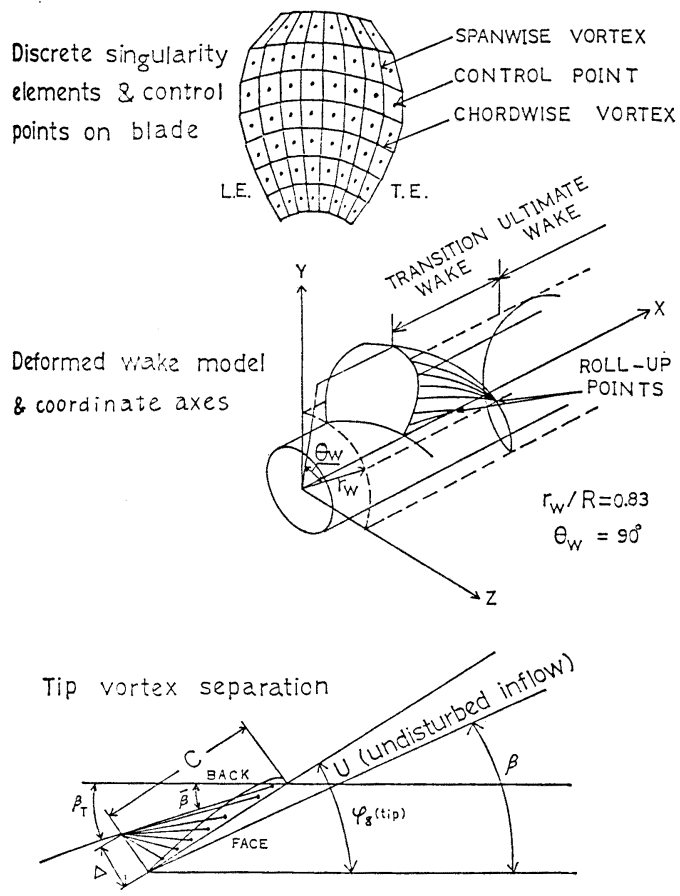

Fig. 1 Illustration of rortex system discrete singularity elements in which the radial interval from the hub to the tip of a propeller is divided into $M$ equal intervals, and the chord from the leading edge to the trailing edge is divided into $N$ equal intervals.

The parameters defining the wake of propeller are

a) Radius of the rolled-up tip vortices, $v_{w}$

b) Angle between trailing edge of blade tip and roll-up point, $\theta_{w}$

c) Pitch angle of outer extremity of transition wake, $\beta_{t}$

d) Pitch angle of ultimate wake tip vortex helix, $\beta_{w}$

For the number of blade division $N$ and $M$ in the present calculation $N=8$ and $M=7$ have been settled after all previous researches. For the parameters defining wake vortex, the roll-up angle $\theta_{w}$ is taken as 90 degree and the slipstream contraction ratio, $r_{w} / R=0.83$ where $R$ is the propeller radius.

On the otherhand, the transition wake pitch angle, $\beta_{t}$ is assumed to be

$$
\beta_{t}=0.4\left[\beta_{(t i p)}+\phi_{g(t i p)}\right]
$$

where

$$
\begin{aligned}
& \beta_{(t i p)}= \text { the mean value of undisturbed inflow } \\
& \text { angle at the tip, } \\
& \phi_{g(t i p)}=\text { geometrical pitch angle at the tip. }
\end{aligned}
$$

The vortex separation at the tip is taken into account as shown in Fig. 1. For the unsteady propeller, with the rotational angle per one-time step " $\delta \theta$ " which is chosen equal to 6 degree the number of divisions of transition wake in the out flow direction $N_{t}=\theta_{w} / \delta \theta=15$. The pitch angle of tip vortex in the ultimate wake is considered equal to the geometrical pitch angle at blade tip $\phi_{g(t i p)}$

\subsection{Circulation in Ship's Wake}

The vortex lattice model for the unsteady propeller is shown in Fig. 2. It was proposed by Kerwin ${ }^{1)}$ and applied to ducted propeller by Yuasa $^{4}$.
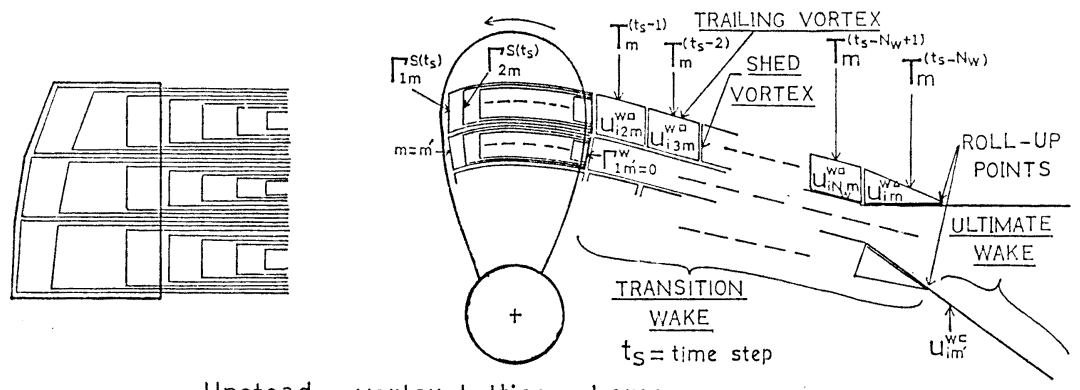

Unsteady vortex lattice scheme

Fig. 2 Vortex lattice model 
In case of steady condition, since there are no effects of shed vortices, the normal component of the total induced velocity at the $i$-th control point on the key blade may be written as

$$
d_{i}=\sum_{k=1}^{K} \sum_{m=1}^{M} \sum_{n=1}^{N} U_{i m n}(k) \Gamma_{n m}^{s}
$$

where $n=1,2, \cdots, N, m=1,2, \cdots, M$, and $K$ is the number of blades. $U_{i n m}(k)$ is the component of induced velocity normal to the mean camber surface at the $i$-th control point due to the horseshoe vortex of unit circulation at the $(n, m)$-th element on the $k$-th blade. The strength of circulation of the horse-shoe vortex is given by that of the spanwise vortex which is designated as $\Gamma_{n m}^{s}$. The boundary value condition is given by

$$
d_{i}=-\boldsymbol{n}_{i}\left(\boldsymbol{V}_{i}{ }^{I}+\boldsymbol{V}_{i}^{q}\right)
$$

where $\boldsymbol{n}_{i}$ is the unit normal vector at the $i$-th control point, $\boldsymbol{V}_{i}^{I}$ is the vector of inflow velocity which is uniform in the steady condition and $\boldsymbol{V}_{i}^{q}$ is the velocity vector induced by source distribution at the $i$-th control point. Solving Eq. (2) under the condition of Eq. (3), $\Gamma_{n m}^{s}$ can be obtained. In calculation of $U_{i n m}(k)$, it should be noticed that the spanwise vortices only in the section of mid-span $\left(m=m^{\prime}\right)$ are preserved in the ultimate wake.

In case of the unsteady condition for the propeller in ship's wake, Kerwin's method has been applied after making some modification to analyze the circulation of vortex in ship's wake into harmonic components. Namely, the harmonic analytical method $^{9)}$ has been adopted which is usually used in the problems of harmonical vibration. In the case of a harmonic wake researched previously in the paper 5), this harmonic analytical method was employed. In the present paper the method is extended to the usual case of ship's wake. The inflow velocity field is assumed to be in $X$-coordinate direction which is taken parallel to the propeller shaft afterwards. The wake velocity field is represented as Fourier series:

$$
w(r, \theta)=w_{0}+\sum_{j=1}^{J}\left(a_{j} \cos j \theta+b_{j} \sin j \theta\right)
$$

where $r$ is a radial distance from the center of propeller shaft and $\theta$ is the angle which is zero vertically upward and positive clockwise when looking downstream. If we assume finite Fourier series up to the $J$-th order, the $X$-component of inflow velocity on the $i$-th control point will be

$$
V_{x}\left(r, \theta_{i}\right)=\sum_{j=0}^{J} V_{x j}(r) \cos \left\{j\left(\theta_{i}-t_{s} \delta \theta-\alpha_{j}(r)\right)\right\}
$$

where $V_{x j}(v)$ is the amplitude of $j$-tin order of Fourier series and $\alpha_{j}(v)$ is the phase lag. The circulation of vortex in unsteady calculation can be determined from a set of the following simultaneous equations

$$
\begin{aligned}
d_{i}= & \sum_{k=1}^{K}\left[\sum_{m=1}^{M} \sum_{n=1}^{N} U_{i n m}^{D_{n}}(k) \Gamma_{n m}^{s\left(t_{s}\right)}(k)\right. \\
& +\sum_{\substack{m=1 \\
\left(m \neq m^{\prime}\right)}}^{M}\left\{\sum_{n_{w=2}}^{N} U_{i n_{w}}^{w \square}(k) T_{m}^{\left(t_{s}-n_{w^{+1}}\right)}(k)\right. \\
& \left.+U_{i m}^{w \Delta}(k) T_{m}^{\left(t_{s}-N_{w^{\prime}}\right)}(k)\right\} \\
& \left.\left.+U_{i m^{\prime}}^{w \sqsubset[}(k) \sum_{n=1}^{N} \Gamma_{n m^{\prime}}^{s\left(t, s^{\prime}\right)}(k)\right]\right]
\end{aligned}
$$

where $t_{s}$ represents time step $\left(t_{s}=\cdots,-2,-1,0\right.$, $1,2, \cdots), T_{m}^{\left(t s^{\prime}\right.}(k)$ is the total circulation around the $m$-th chordwise panel at time $t_{s}$. The normal components of induced velocities of vortex rings, $U_{i n m}^{\square}(k)$ and $U_{i n_{w} m}^{w \square}(k)$ are given by

$$
\begin{aligned}
& U_{i n m}^{\square}(k)=\left\{U_{i n m}^{s}(k)-U_{i 1 m}^{w}(k)\right\} \\
& +\sum_{l=n}^{N}\left\{U_{i l(m+1)}^{c}(k)-U_{i l m}^{c}(k)\right\} \\
& \left.\begin{array}{rl}
U_{i n_{w m}}^{w \square}(k)= & U_{i\left(n_{\left.w^{-1}\right) m}\right.}^{w}(k)+U_{i\left(n_{w}-1\right)(m+1)}^{t}(k) \\
& -U_{i\left(n_{\left.w^{-1}\right) m}^{t}\right.}^{t}(k)-U_{i n_{w} m}^{w}(k)
\end{array}\right)
\end{aligned}
$$

where superscript $s, c, w, t=$ spanwise, chordwise, shed and trailing vortices respectively.

$U_{i m}^{w \Delta}(k)$ is that of the triangle vortex ring at the end of the transition vortex wake, which can be calculated by eliminating the last term of shed vortex in the formula of $U_{i n_{w} m}^{w \square}(k)$.

In correspondence to the harmonic components of ship's wake which are given by Fourier series as Eq. (4) or Eq. (5), the circulations of spanwise vortex and vortex ring in the transition wake of vortex, $\Gamma_{n m}^{s\left(t_{s}\right)}(k)$ and $T_{m}^{\left(t_{s}\right)}(k)$ can be also decomposed into a steady term and unsteady terms which are represented by Fourier series, using coefficients $g_{0 n m}, g_{1 n m}^{(j)}, g_{2 n m}^{(j)}$, as follows:

$$
\begin{gathered}
\Gamma_{n m}^{s\left(t_{s}\right)}(k)=\bar{\Gamma}_{n m}^{s}(k)+\tilde{\Gamma}_{n m}^{s\left(t_{s}\right)}(k) \\
\bar{\Gamma}_{n m}^{s}(k)=g_{0 m n} \quad \text { (steady term) } \\
\tilde{\Gamma}_{n m}^{s\left(t_{s}\right)}(k)=\sum_{j=1}^{J}\left[g_{1 n m}^{(j)} \cos \left\{j\left(\theta_{n m}+\delta_{k}-t_{s} \delta \theta\right)\right\}\right. \\
\left.+g_{2 n m}^{(j)} \sin \left\{j\left(\theta_{n m}+\delta_{k}-t_{s} \delta \theta\right)\right\}\right] \\
\quad \text { (unsteady terms) } \\
T_{m}^{\left(t_{s}\right)}(k)=\bar{T}_{m}(k)+\tilde{T}_{m}^{\left(t_{s}\right)}(k) \\
\bar{T}_{m}(k)=\sum_{n=1}^{N} g_{0 n m} \quad \text { (steady term) }
\end{gathered}
$$




$$
\begin{aligned}
\tilde{T}_{m}^{\left(t_{s}\right)}(k)= & \sum_{j=1}^{J} \sum_{n=1}^{N}\left[g_{1 n m}^{(j)} \cos \left\{j\left(\theta_{n m}+\delta_{k}-t_{s} \delta \theta\right)\right\}\right. \\
& \left.+g_{2 n m}^{(j)} \sin \left\{j\left(\theta_{n m}+\delta_{k}-t_{s} \delta \theta\right)\right\}\right]
\end{aligned}
$$

(unsteady terms)

where

$$
\delta_{k}=2 \pi(k-1) / K, \quad(k=1,2, \cdots, K) .
$$

The boundary condition in this unsteady case can be written as follows, substituting Eq. (5) into Eq. (3).

$$
\begin{aligned}
d_{i}= & -n_{i x} V_{x 0}\left(v_{i}\right) \\
& -n_{i x} \sum_{j=1}^{J}\left[V_{x j}\left(v_{i}\right) \cdot \cos \left\{j\left(\theta_{i}-t_{s} \delta \theta-\alpha_{j}\left(v_{i}\right)\right\}\right]\right. \\
& -\boldsymbol{n}_{i} \boldsymbol{V}_{i}^{\boldsymbol{q}}
\end{aligned}
$$

The first and the last terms of Eq. (15) are steady and the second summation terms are unsteady. Substituting Eqs. (8)-(13) into Eq. (6) and considering the boundary condition (15), a set of simultaneous equations with respect to $g_{0 \mathrm{~nm}}$, $g_{1 n m}^{(j)}, g_{2 n m}^{(j)}$ are deduced as follows.

Steady part:

$$
\begin{aligned}
\sum_{k=1}^{K}\left[\sum _ { m = 1 } ^ { M } \sum _ { n = 1 } ^ { N } g _ { 0 n m } \left\{U_{i n m}^{\square}(k)+\sum_{n=2}^{N_{w}} U_{i n_{w} m}^{w \square}(k)\right.\right. \\
\left.+U_{i m}^{w \Delta}(k)\right\}+\sum_{n=1}^{N} g_{0 n m^{\prime}} \\
\left.\quad \times\left\{U_{i n m^{\prime}}^{\square}(k)+U_{i m^{\prime}}^{w \sqsubset}(k)\right\}\right] \\
=-n_{i x} V_{x 0}\left(v_{i}\right)-\boldsymbol{n}_{i} \boldsymbol{V}_{i}^{q}
\end{aligned}
$$

Unsteady part:

$$
\begin{aligned}
& \sum_{k=1}^{K}\left\langle\sum _ { \substack { m = 1 \\
( m \neq m ^ { \prime } ) } } ^ { M } \sum _ { n = 1 } ^ { N } \sum _ { j = 1 } ^ { J } g _ { 1 n m } ^ { ( j ) } \left[ U_{i n m}^{D}(k)\right.\right. \\
& \cdot \cos \left\{j\left(\theta_{n m}+\delta_{k}-t_{s} \delta \theta\right)\right\} \\
& +\sum_{n_{w}=2}^{N_{w}} U_{i n_{w}}^{w q^{m}}(k) \cdot \cos \left\{j \left(\theta_{n m}+\delta_{\kappa}\right.\right. \\
& \left.\left.-\left(t_{s}-n_{w}+1\right) \delta \theta\right)\right\}+U_{i m}^{w \Delta}(k) \\
& \left.\cdot \cos \left\{j\left(\theta_{n m}+\delta_{k}-\left(t_{s}-N_{w}\right) \delta \theta\right)\right\}\right] \\
& +\sum_{n=1}^{N} \sum_{j=1}^{J} g_{1 n m^{\prime}}^{(j)}\left[U _ { i n m ^ { \prime } } ^ { D ^ { \prime } } ( k ) \cdot \operatorname { c o s } \left\{j \left(\theta_{n m^{\prime}}+\delta_{k}\right.\right.\right. \\
& \left.\left.\left.-t_{s} \delta \theta\right)\right\}+U_{i m^{\prime}}^{w \sqsubset}(k) \cdot \cos \left\{j\left(\theta_{n m^{\prime}}+\delta_{\kappa}-t_{s} \delta \theta\right)\right\}\right] \\
& +\sum_{\substack{m=1 \\
\left(m \neq m^{\prime}\right)}}^{M} \sum_{n=1}^{N} \sum_{j=1}^{J} g_{2 n m}^{(j)}\left[U_{i n m}^{D}(k)\right. \\
& \cdot \sin \left\{j\left(\theta_{n m}+\delta_{k}-t_{s} \delta \theta\right)\right\}+\sum_{n_{w}=2}^{N_{w}} U_{i n_{w} m}^{w \square}(k) \\
& \cdot \sin \left\{j\left(\theta_{n m}+\delta_{k}-\left(t_{s}-n_{w}+1\right) \delta \theta\right)\right\} \\
& \left.+U_{i m}^{w \Delta}(k) \cdot \sin \left\{j\left(\theta_{n m}+\delta_{k}-\left(t_{s}-N_{w}\right) \delta \theta\right)\right\}\right] \\
& +\sum_{n=1}^{N} \sum_{j=1}^{J} g_{2 n m^{\prime}}^{(j)}\left[U _ { i n m ^ { \prime } } ^ { D ^ { \prime } } ( k ) \cdot \operatorname { s i n } \left\{j \left(\theta_{n m^{\prime}}\right.\right.\right.
\end{aligned}
$$

$$
\begin{aligned}
& \left.\left.+\delta_{k}-t_{s} \delta \theta\right)\right\}+U_{i m^{\prime}}^{w \sqsubset}(k) \\
& \left.\left.\cdot \sin \left\{j\left(\theta_{n m^{\prime}}+\delta_{\kappa}-t_{s} \delta \theta\right)\right\}\right]\right\rangle \\
= & \sum_{j=1}^{J}\left[-n_{x} V_{x j}\left(v_{i}\right) \cdot \cos \left\{j\left(\theta_{i}-t_{s} \delta \theta-\alpha_{j}\left(v_{i}\right)\right)\right\}\right]
\end{aligned}
$$

The equation (17) may be solved individually for each harmonic mode of the $j$-th order. Each equation for the single harmonic mode is the same as the equation (A-2) in the reference 5).

\subsection{Hydrodynamic Forces}

The force acting on the blade camber surface is composed of the following four components:

A) The force acting on an element of the source sheet:

This force can be calculated based on Lagally's theorem as follows,

$$
\boldsymbol{F}_{i p}^{(L)}=-\rho \Delta l_{i p} \boldsymbol{V}_{i p} Q_{i p}
$$

where $\rho$ is fluid density, $Q_{i p}$ the density of source and $\boldsymbol{V}_{i p}$ is the resultant velocity vector on the $(i, p)$-th segment whose length is $\Delta l_{i p} . \quad Q_{i p}$ is approximately determined by

$$
Q_{i p}=\int_{\xi_{i}}^{\xi_{i+1}} U \frac{d t_{h}(\xi)}{d \xi} d \xi=U\left\{t_{h}\left(\xi_{i+1}\right)-t_{h}\left(\xi_{i}\right)\right\}
$$

where $U$ is an undisturbed inflow velocity, $\xi$ is the chord direction coordinate and $t_{h}$ is blade thickness function. $\boldsymbol{V}_{\boldsymbol{i p}}$ is the resultant velocity vector at the mid point of the segment $\Delta l_{i p}$, which can be gotten from the following equation

$$
\begin{aligned}
& \boldsymbol{V}_{i p}=\sum_{k=1}^{K}\left[\sum_{m=1}^{M} \sum_{n=1}^{N} \boldsymbol{U}_{i p n m}^{p_{n}}(k)\left\{\bar{\Gamma}_{n m}^{s}+\tilde{\Gamma}_{n m}^{s\left(t_{s}\right)}(k)\right\}\right. \\
& +\sum_{\substack{m=1 \\
\left(m=m^{\prime}\right)}}^{M}\left\{\sum_{n w=2}^{N_{w}} \boldsymbol{U}_{i p{ }_{w w m}}^{w g_{w}}(k)\left(\bar{T}_{m}+\tilde{T}_{m^{\left(t_{s}-n_{w}+1\right)}}(k)\right)\right. \\
& \left.+\boldsymbol{U}_{i p m}^{w \Delta}(k)\left(\bar{T}_{m}+\tilde{T}_{m}^{\left(t_{s}-N_{w}\right)}(k)\right)\right\} \\
& \left.+\boldsymbol{U}_{i p m^{\prime}}^{w[}(k) \sum_{n=1}^{N} \Gamma_{\left.n m^{\prime}, s\right)}^{s(t, s)}(k)\right] \\
& +\left[V_{x 0}+\sum_{j=1}^{J} V_{x j} \cos \left\{j\left(\theta_{i p}-t_{s} \delta \theta-\alpha_{j}\right)\right\}\right] \boldsymbol{i} \\
& +\left(-2 \pi n r_{i p} \sin \theta_{i p}\right) \boldsymbol{j} \\
& +\left(2 \pi n r_{i p} \cos \theta_{i p}\right) \boldsymbol{k}
\end{aligned}
$$

where $U_{i p n m}$ is the induced velocity vector on the mid-point of $(i, p)$-th segment due to the vortex of a unit circulation at the $(n, m)$-th segment, and $\boldsymbol{i}, \boldsymbol{j}, \boldsymbol{k}$ are unit vectors in $x, y, z$ directions respectively.

B) The force acting on an element of the vortex sheet.

This force can be obtained from the KuttaJoukowski theorem as follows: 


$$
\boldsymbol{F}_{i p}^{(K J)}=\rho \Delta l_{i p} \cdot \boldsymbol{V}_{i p} \times \Gamma_{i p}
$$

where $\boldsymbol{\Gamma}_{i p}$ is the vector representation of the circulation on the $(i, p)$-th spanwise or chordwise vortex, which is assumed to be located in the $(m, n)$-th element,

spanwise vortex:

$$
\begin{aligned}
\Gamma_{i p}^{s\left(t_{s}\right)}= & \left\langle g_{0 n m}+\sum_{j=1}^{J}\left[g_{1 n m}^{(j)} \cos \left\{j\left(\theta_{i p}+\delta_{k}-t_{s} \delta \theta\right)\right\}\right.\right. \\
& \left.\left.+g_{2 n m}^{(j)} \sin \left\{j\left(\theta_{i p}+\delta_{k}-t_{s} \delta \theta\right)\right\}\right]\right\rangle \\
& \times \frac{\Delta \boldsymbol{l}_{i p}^{s}}{\left|\Delta \boldsymbol{l}_{i p}^{s}\right|}
\end{aligned}
$$

chordwise vortex:

$$
\Gamma_{i p}^{c\left(t_{s}\right)}=\left\{\sum_{l=1}^{n}\left(\Gamma_{l(m-1)}^{s}-\Gamma_{l m}^{s}\right)\right\} \frac{\Delta \boldsymbol{l}_{i p}^{c}}{\left|\Delta \boldsymbol{l}_{i p}^{c}\right|}
$$

C) Viscous frictional drag force:

This force is assumed to act in the chordwise direction and may be determined as:

$$
\boldsymbol{F}_{i p}^{(F)}=\frac{1}{2} \rho C_{f} \Delta A_{i p}\left|\boldsymbol{V}_{i p}\right| \boldsymbol{V}_{i p}
$$

drag coefficient $C_{f}$ can be determined from Prandtl-Schlicting formula such as

$$
C_{f}=\left(1+t_{m} / c\right)\left\{0.455 /\left(\log _{10} R_{e}\right)^{2.58}\right\}
$$

where $t_{m}$ is maximum blade thickness, $c$ is the chord length of blade section and $R_{e}$ is the Reynolds number of the blade section. $\Delta A_{i p}$ is the area of the $(i, p)$-th element.

D) The force proportional to the time derivative of circulation.

This time derivative term arises from the unsteady term of momentum equation due to the time change rate of pressure difference between upper and lower surfaces.

This force may be determined such as

$$
\boldsymbol{F}_{i p}^{(T)}=\rho \boldsymbol{n}_{i p} \frac{\partial}{\partial t}\left[\sum_{l=1}^{i} \Gamma_{l p}^{s}\right] \frac{\Delta \gamma_{i p}}{\cos \varepsilon} \Delta c
$$

where $\boldsymbol{n}_{i p}$ is unit normal vector at the mid-point of the $(i, p)$-th spanwise segment whose radial length is $\Delta r_{i p}, \varepsilon$ is the rake angle of the segment and $\Delta c$ is the chord length of $\Delta A_{i p}(=\Delta c \cdot \Delta r)$ $\cos \varepsilon)$.

\section{Results of Calculations and Experiments}

We have chosen a marine propeller MAU5-66 whose expanded area ratio is 0.6649 and pitch

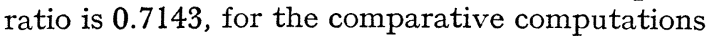
by means of present method (P.M.) and Koyama's method (K.M.). The calculations of thrust and torque have been carried out for two model propellers whose diameters are $0.1167 \mathrm{~m}$ and $0.175 \mathrm{~m}$, and the measurements only for $0.175 \mathrm{~m}$ propeller.

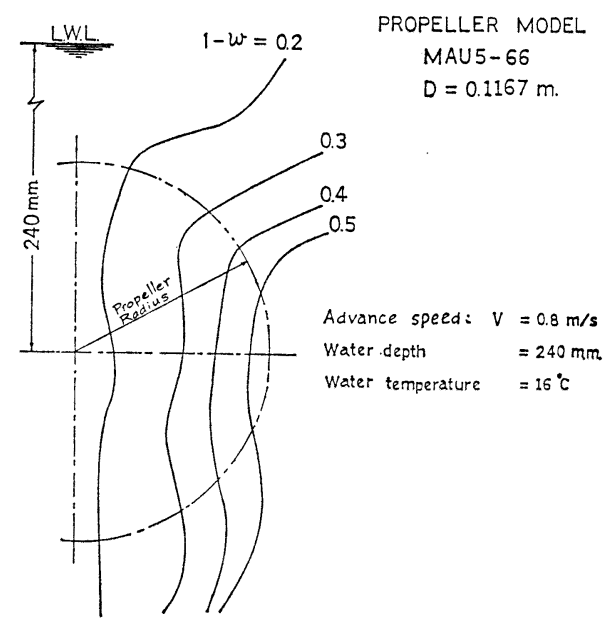

Fig. 3 Contour line of $1-w=V_{x} / V$ (Type A)

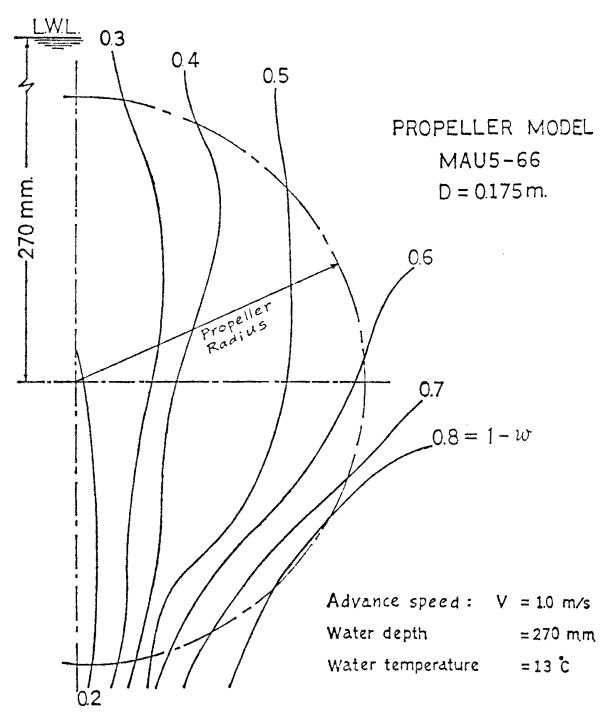

Fig. 4 Contour line of $1-w=V x / V$ (Type B)

\subsection{Ship's Wake}

Employing the method of wire mesh screen, we have gotten two different types of wake pattern. Figs. 3 and 4 illustrate the two types. Type A in Fig. 3 simulates a wake pattern of the hull form with U-shape framelines and Type B in Fig. 4 simulates one of the hull form with Vshape framelines. The harmonic analysis has been done into Fourier series on every tenth of the radius of the propeller disk, whose diameter is $0.1167 \mathrm{~m}$ for Type A wake and is $0.175 \mathrm{~m}$ for Type B wake. The highest mode of harmonic components has been taken as eight for Type A, namely $J=8$ in Eq. (4), and as ten for Type B, namely $J=10$ in Eq. (4). The coefficients of Fourier series, $a_{j}$ of Eq. (4), are shown in Table 1 
Table 1 Coefficients of Fourier series of ship's wake

$$
w(\theta)=w_{0}+\sum_{j=1}^{J}\left(a_{j} \cos j \theta+b_{j} \sin j \theta\right), b_{j}=0
$$

\begin{tabular}{|c|c|c|c|c|c|c|c|}
\hline \multirow{2}{*}{$\frac{r / R}{\text { Wake }}$} & 0.3 & \multicolumn{2}{|l|}{0.5} & \multicolumn{2}{|c|}{0.7} & \multicolumn{2}{|c|}{0.9} \\
\hline & Type A Type B & Type A 1 & Type B & Type A & Type B & Type A & Type B \\
\hline$a_{1}$ & $0.0001 \quad 0.0011$ & 0.0002 & 0.0141 & 0.0114 & 0.0299 & 0.0227 & 0.0872 \\
\hline$a_{2}$ & $0.0321 \quad 0.0839$ & 0.0514 & 0.1283 & 0.1070 & 0.1284 & 0.1594 & 0.1412 \\
\hline$a_{3}$ & $0.0001-0.0188$ & $0.0002-c$ & -0.0182 & -0.0034 & -0.0193 & -0.0108 & -0.0664 \\
\hline$a_{4}$ & $0.0029-0.0010$ & 0.0071 & 0.0248 & 0.0092 & 0.0485 & 0.0039 & 0.0863 \\
\hline$a_{5}$ & $0.0001-0.0052$ & 0.0002 & 0.0235 & 0.0036 & -0.0303 & 0.0084 & -0.0232 \\
\hline$a_{6}$ & $0.0035 \quad 0.0115$ & 0.0055 & 0.0006 & 0.0075 & 0.0166 & 0.0035 & 0.0323 \\
\hline$a_{7}$ & $0.0001-0.0065$ & $0.0002-$ & -0.0112 & 0.0021 & -0.0149 & 0.0049 & -0.0280 \\
\hline$a_{3}$ & $0.0004-0.0060$ & 0.0031 & 0.0119 & 0.0047 & 0.0057 & 0.0026 & 0.0022 \\
\hline$a_{9}$ & $-\quad-0.0035$ & - & 0.0055 & - & -0.0123 & - & -0.0088 \\
\hline$a_{10}$ & 0.0015 & - & 0.0002 & - & 0.0042 & - & 0.0194 \\
\hline$w_{0}$ & $0.7919 \quad 0.7055$ & 0.7529 & 0.6273 & 0.7019 & 0.5855 & 0.6607 & 0.4956 \\
\hline
\end{tabular}

at $0.3 R, 0.5 R, 0.7 R$ and $0.9 R$, where all $b_{j}$ values become zero because of the symmetry of the wake pattern. One can find in Table 1 that the predominant term is $a_{2}$ for Type A and also for Type B. The term of $a_{4}$ seems to have a secondary effect in case of Type B. Other terms are of smaller order.

\subsection{Thrust and Torque in Ship's Wake}

At first, the thrust and the torque per one blade (key blade) have been calculated by P.M. and by K.M. The results in case of Type A wake are shown in Figs. 5 and 6 , and those in case of Type B wake in Figs. 7 and 8. The amplitude of fluctuation of either thrust or torque calculated by P.M. is much greater than that by K.M. Particularly the former attains $250 \%$ of the latter in the torque in Type B wake. This is a remarkable effect of amplification for the 2nd and the 4th harmonic wake fluctuation, which was pointed out in the reference 5) or 6). The calculation by P.M. shows the phase lag of about

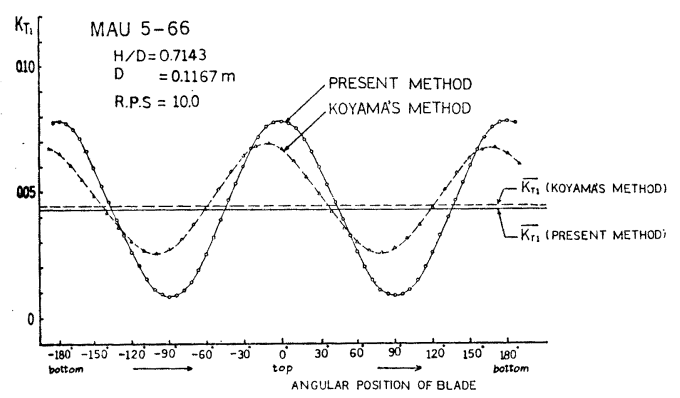

Fig. 5 Thrust fluctuation of one blade in Type A wake
20 degrees from the calculation by K.M. in case of Type A wake, but both have almost the same phase.

Next, thrusts and torques of all blades have been summed up respectively considering the phase shift of each blade. The calculated results are shown in Figs. 9-12. The $175 \phi$ model propeller has only been tested for measuring thrust and torque in the wake of Type B. The recorded data have been numerically treated to cut off fluctuating components of higher frequency than $100 \mathrm{HZ}$ that corresponds to the twice of the blade frequency as the noise.

The measured results are found a little higher than the calculations on the average in both thrust (Fig. 11) and torque (Fig. 12). This fact may be considered due to a little increase of wake interacted by the propeller action. It can be pointed out in Fig. 11 that the fluctuation of thrust by P.M. calculation shows a good agreement with that of experiment. It is composed of

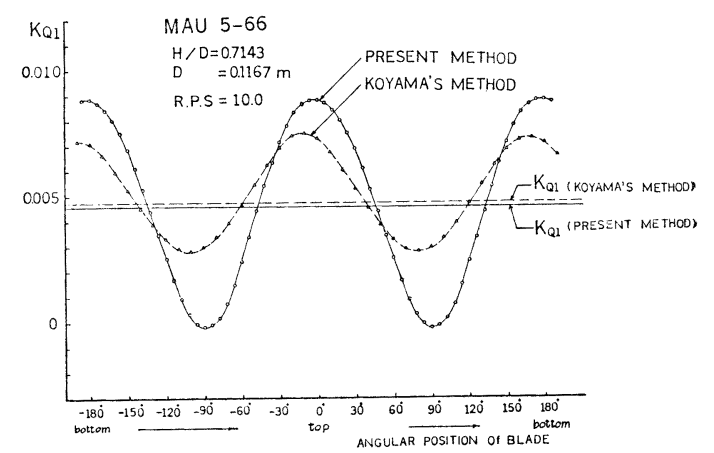

Fig. 6 Torque fluctuation of one blade in Type A wake 


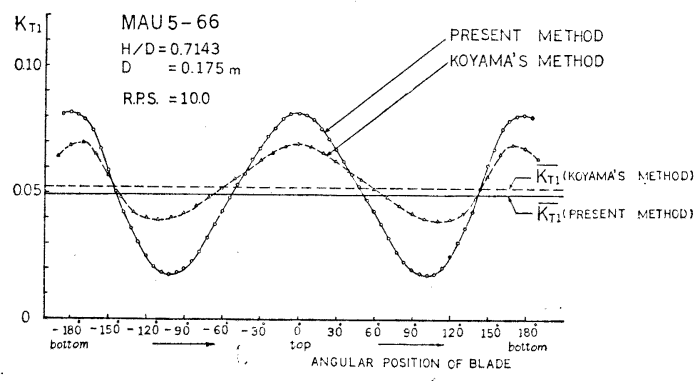

Fig. 7 Thrust fluctuation of one blade in Type B wake

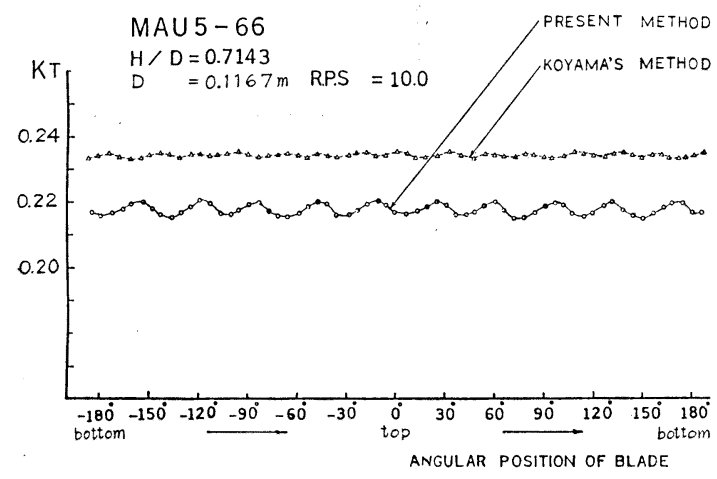

Fig. 9 Thrust fluctuation of all blades in Type A wake

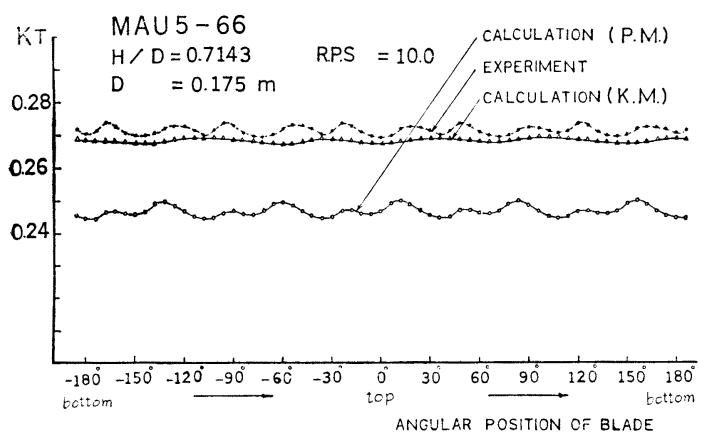

Fig. 11 Thrust fluctuation of all blades in Type B wake

two dominant harmonic components, one is the blade frequency term of the $5 \theta$ mode and the other is the double blade frequency term of the $10 \theta$ mode, but, in Fig. 12, the fluctuation of torque by P.M. calculation exhibits an excess estimation of the harmonic component of the $5 \theta$ mode along with less magnitude of the $10 \theta$ mode and also a phase advance in comparison with the measured torque curve.

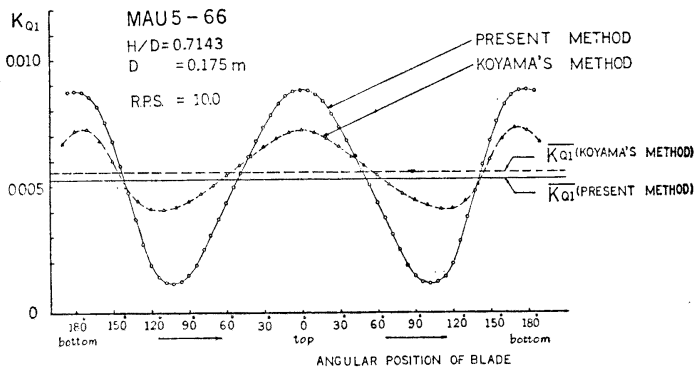

Fig. 8 Torque fluctuation of one blade in Type B wake

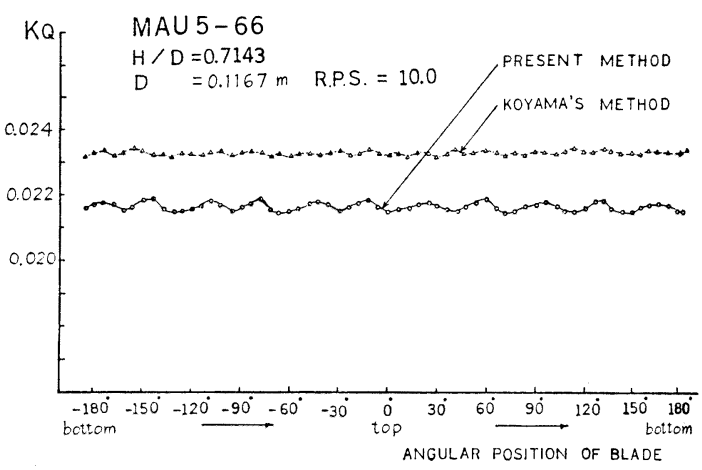

Fig. 10 Torque fluctuation of all blades in Type A wake

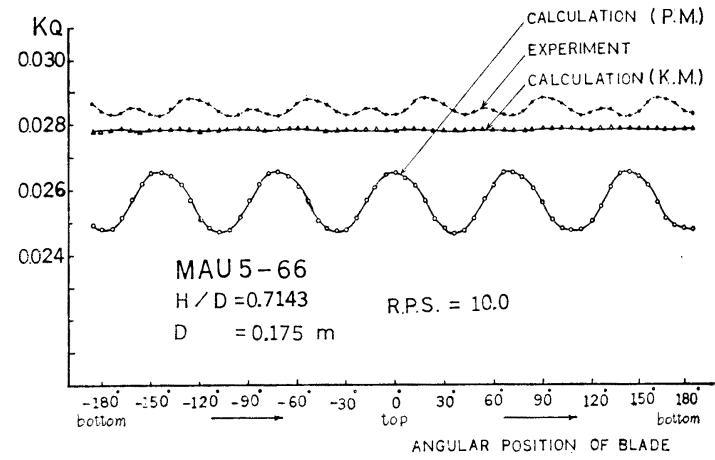

Fig. 12 Torque fluctuation of all blades in Type B wake

The K.M. calculation is found to predict less fluctuations of thrust and torque not only in case of Type B wake shown in Figs. 11 and 12 but also in case of Type A wake shown in Figs. 9 and 10, though the mean lines of K.M. are close to those of experiment.

The relative rotative efficiency $\eta_{R}$ has been evaluated in order to investigate the non-linear effect of the non-uniformity of ship's wake, Type 


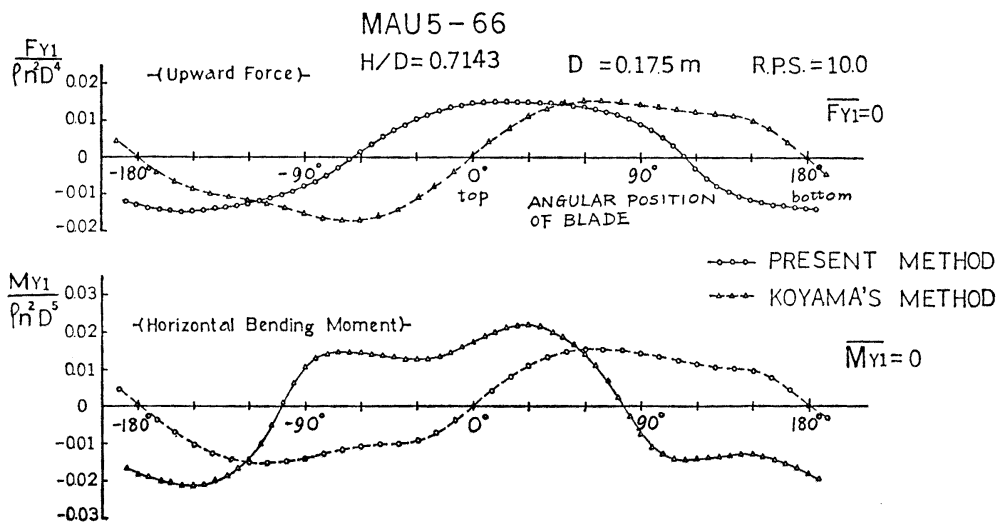

Fig. 13 Bearing force (upward) and moment (horizontal) in Type B wake

Table 2 Relative rotative efficiency, $\eta_{R}$

\begin{tabular}{l|c|c}
\hline \multicolumn{1}{c|}{ Wake } & Type A & Type B \\
\hline Calculation by P.M. & 1.079 & 1.067 \\
Experiment & - & 1.057 \\
\hline
\end{tabular}

A and Type B. The considerable high values of $\eta_{R}$ have been obtained as seen in Table 2. The difference between calculation and experiment in case of Type B looks so little that $\eta_{R}$ may be estimated within enough accuracy by P.M. calculation. The calculated $\eta_{R}$ for Type A reveals a little higher efficiency of the propeller in the wake pattern of the $U$-shape stern.

\subsection{Bearing Forces and Moments}

For examples of bearing forces and moments calculated by P.M. and K.M., the upward force and the horizontal moment per one blade (key blade) which are denoted by $F_{y_{1}}$ and $M_{y_{1}}$ respectively are shown in Fig. 13. This example is the calculation in case of Type B wake, where the harmonic component of $\cos 2 \theta$ mode is dominant and that of $\cos 4 \theta$ mode is about a quarter of the $\cos 2 \theta$ component and others are negligible as seen in Table 1. Therefore, both $F_{y 1}$ and $M_{y_{1}}$ can be considered to consist of harmonic components of $(2 \pm 1) \theta$ mainly and $(4 \pm 1) \theta$ secondarily. The difference between P.M. and K.MI. is so much in phase property as to be given an attention to in both cases of $F_{y 1}$ and $M_{y 1}$. The main reason will be the influence of the $3 \theta$ and $5 \theta$ components due to the non-linear effect of $a_{2} \cos 2 \theta$ wake component taken into consideration in P.M. The summation of $F_{y 1}$ or $M_{y 1}$ for all blades will certainly become small in this case for the five bladed propeller like MAU5-66, because the $4 \theta$ and $6 \theta$ harmonic components of the wake which are main contributors to bearing forces and moments are not dominant.

\section{Conclusions}

Comparing the unsteady calculation by the present method of vortex lattice model with that of Koyama's method, it has been found that there exists quite difference in the lift property that the present method has a tendency to increase the amplitude of fluctuation of thrust and torque.

Comparing the unsteady calculations of thrust and torque by the present method with the experimental results in a ship's wake, it has been made clear that the effect of the higher order harmonic component due to the non-linearity predicted by P.M. is true.

The bearing forces and moments calculated by the present method differs much in phase from that by Koyama's method. This also is owing to non-linear effects taken into consideration in the present method.

\section{Acknowledgements}

The authors greatly appreciate Mr. H. Funaki's cooperative works in analyses and experiments and Mr. M. Ando's advices in computations. All computations have been carried out using M$280 \mathrm{H} / \mathrm{M}-200 \mathrm{H}$ of Tokyo University and M-240H of Yokohama National University.

\section{References}

1) Kerwin, J. E. and Lee, C. S.: Prediction of Steady and Unsteady Marine Propeller Performance by Numerical Lifting Surface Theory, Transactions of Society of Naval Architects and Marine Engineers, Vol. 86, 1978.

2) Kerwin, J. E.: Flow Field Computations for Non-Cavitating and Cavitating Propel- 
lers, Office of Naval Research, U.S.A., 1982.

3) James, R. E.: On the Remarkable Accuracy of the Vortex Lattice Method, Computer Method in Applied Mechanics and Engineering 1, Holland, 1972.

4) Yuasa, H.: Application of Numerical Lifting-Surface Theory on Steady Performance of Propeller/Duct System, Journal of Society of Naval Architects of Japan, Vol. 147, 1980.

5) Ikehata, M., Ando, M. and Maruo, H.: The Analysis of Unsteady Characteristics of Marine Propeller in Harmonic Wake by Vortex Lattice Lifting-Surface Model, Journal of Society of Naval Architects of Japan, Vol. 153,1983.

6) Maruo, H., Ikehata, M. and Ando, M.: Theoretical Prediction of Unsteady Propel- ler Characteristics in the Non-Uniform Wake Field, 15th Symposium on Naval Hydrodynamics, Hamburg, September, 1984.

7) Koyama, K.: A Numerical Method for Propeller Lifting Surface in Non-Uniform Flow and Its Application, Journal of the Society of Naval Architects of Japan, Vol. 137, 1975.

8) Koyama, K.: On Application of the Lifting Surface Theory to Marine Propellers, 13th Symposium on Naval Hydrodynamics, Tokyo, October, 1980.

9) Hanaoka, T.: Hydrodynamics of an Oscillating Screw Propeller, 4th Symposium of Naval Hydrodynamics, Washington D.C., 1962. 